\title{
Paper
}

\section{A New Lighting Communication System for Audio Signal with White LED}

\author{
Shun LIU*, Atsushi MINATO*, Satoru OZAWA* and Masao NAKAGAWA* \\ *Ibaraki University, Graduate School of Science and Engineering, Institute of Applied Beam Science \\ **Keio University, Department of Information and Computer Science
}

Received August 28, 2006, Accepted February 15, 2007

\begin{abstract}
Light Emitting Diode (LED) is expected as the lighting source of next generation because they are long life, small size, and low power consumption, etc. In previous paper, we proposed a simple visible-light transmission system for sound using white LED. However, the flicker of light is sensed if the transmitted sound signal has the low frequency component. In this study, we propose improved modulation technique to overcome the flicker. LED can transmit audio signal while it is used for lighting source. We verified that it is possible to communicate with the received luminous flux of $1.1 \times 10^{-5} \mathrm{Im}$ without ficker. We also evaluated the effects of interference by plural LEDs.
\end{abstract}

KEYWORDS: white LED, lighting, luminous Intensity, luminous flux, audio signal, AM radio

\section{Introduction}

Light Emitting Diode (LED) is widely used as the lighting source of next generation such as room lights, car lights, traffic signals and street lights ${ }^{1}$. Moreover, NEDO (New Energy and industrial Technology Development Organization) is aiming to develop white LED for lighting of $120 \mathrm{~lm} / \mathrm{w}$ in "The Light for the 21st Century" National Project. It is considered that LED becomes a lighting source instead of a fluorescent lamp in the future ${ }^{2)}$.

On the other hand, electromagnetic wave communication, what is widely used at present, has following restrictions. Electromagnetic wave cannot be freely used for wireless communication by legal limitations. Electromagnetic wave communication cannot be used in hospital because the electromagnetic wave affects precision machines. In a building, underground or a tunnel, electromagnetic wave of long wavelength cannot propagate.

However, these problems can be solved by visible optical communication ${ }^{3)-7}$. In previous paper, we reported visiblelight communication system composed of simple transmitter with LED and receiver using Amplitude Modulation (AM) radio 8). However a flicker is felt when audio signal has a component of low frequency, because the output power of LED changes with the power of the audio signal. In this study, this technique is improved. Sound signal was transmitted using white LED without flicker.

\section{Communication method}

Figure 1 shows the concept of transmitting system reported in the previous paper. The transmitter consists of a microcomputer, a square-wave oscillator of AM frequency and a logical AND device. Sound signal is converted to Pulse Width Modulation (PWM) signal. An oscillator generates a square wave of AM frequency. The logical product of the PWM and the square wave modulates a LED. This signal has the component of AM frequency and it is proportional to the intensity of input sound signal. Therefore an AM receiver can reproduce the sound converting the light to the radio wave.

Figure 2 shows the concept of receiver where the light signal is transformed to the photon current by photodiode and the photon current is amplified by a transistor. Figure 3 shows the photograph of receiving system. AM radio is placed in the coil. Enameled wire was rolled 100 times. The alternating component of current is transformed to radio wave in the coil. The electromagnetic wave is received by the AM radio. It is not preferable to replace the circuit of the photodiode and the transistor with the phototransistor, because the response of phototransistor is not good at high frequency. The power supply of the receiving circuit is $3-5 \mathrm{~V}$.

The modulation method was improved to remove the flicker of LED. The transmitting circuit was replaced by single microcomputer. Attiny 26 microcomputer was used for the transmitter, because it has analog-to-digital (AD) converter 9). The system clock is $16 \mathrm{MHz}$. Figure 4 shows the flowchart of program of the microcomputer. Figure 5 shows an example of modulation signal.

(1) At first the square-wave oscillation of AM frequency is prepared. 
(2) The input sound signal is $\mathrm{AD}$ converted. The resolution of $\mathrm{AD}$ converter is $8 \mathrm{bit}$. Here we define the $\mathrm{AD}$ converted value as $\mathrm{I}_{\text {on. }}$ The complement of $\mathrm{I}_{\text {on }}$ is calculated $\left(\mathrm{I}_{\text {offif }}\right)$. The total of $\mathrm{I}_{\text {on }}$ and $\mathrm{I}_{\text {off }}$ becomes 255 .

(3) Within the period of $I_{\text {on }}$ the LED is modulated by the square wave of AM frequency. This period corresponds to the $\mathrm{T}_{\text {on }}$ in Fig. 5.

(4) Within the period of $\mathrm{I}_{\text {off }}$ the LED is turned off. This period corresponds to the $\mathrm{T}_{\text {off }}$ in Fig. 5.

(5) The input signal is $\mathrm{AD}$ converted again.

(6) Within the period of $\mathrm{I}_{\text {on }}$ the LED is modulated by the square wave of AM frequency.

(7) Within the period of $I_{\text {off }}$ the LED is turned on.

(8) Return to (2).

The period, $\mathrm{T}$, is $48 \mu \mathrm{sec}$ in Fig. 5 . In the improved technique, LED is turned on alternately while the period


cycle (2T). Within the period of $\mathrm{T}_{\mathrm{on}}$, the duty of LED is $50 \%$. LED is turned off within the period of the first $\mathrm{T}_{\text {off, }}$ and

\section{Square Wave $(600-1600 \mathrm{kHz})$}


Figure 1 Concept of transmitting system reported in the previous paper

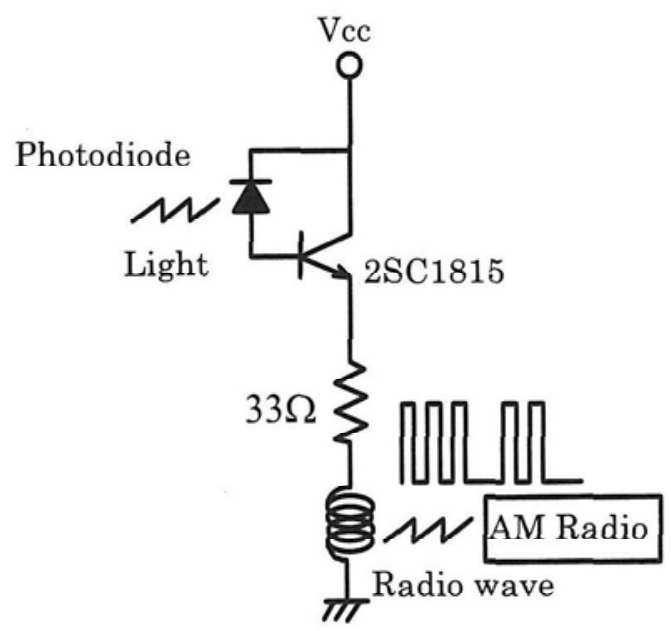

Figure 2 Concept of receiver

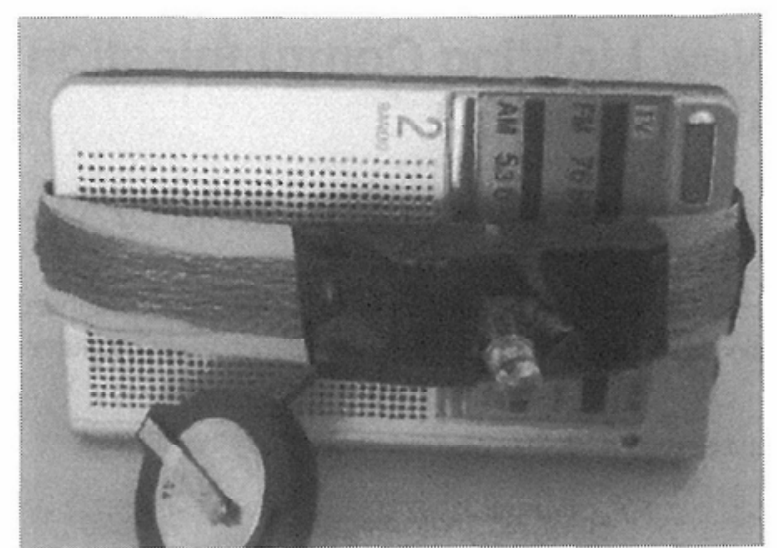

Figure 3 Photograph of receiver

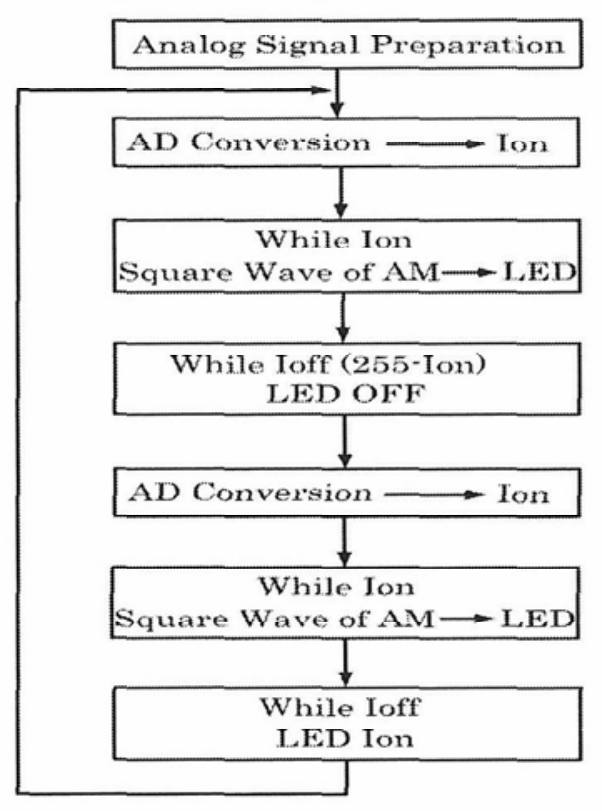

Figure 4 Flowchart of program of the microcomputer

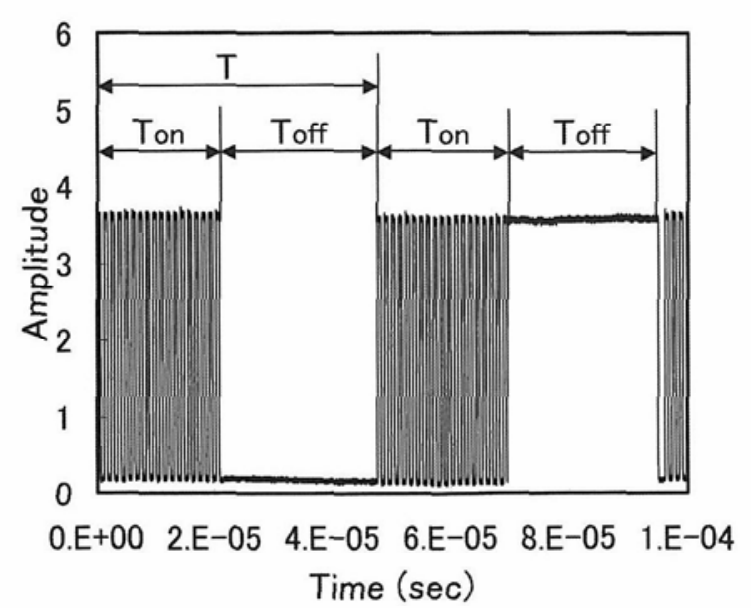

Figure 5 Example of modulation signal 
LED is turned on within the period of the second $T_{\text {off: }}$ The period of one cycle ( $2 \mathrm{~T})$ is much smaller than the period of the change of the audio signal. In this case, the first $\mathrm{T}_{\text {off }}$ and the second $\mathrm{T}_{\text {off }}$ are nearly equal. Therefore the duty of LED becomes $50 \%$ in one cycle (2T). In general, the maximum flicker frequency that people's eyes can perceive is tens of Hertz. The modulation frequency of LED is much higher than this value. Therefore people's eyes detect only constant illumination of LED with the duty of $50 \%$, and the fluctuation cannot be detected.

\section{Stability of LED output}

Communication experiment was carried out using a white LED and a photodiode. NSPW500BS (Nichia) LED was used. The diameter is $5 \mathrm{~mm}$ and the luminous intensity is $9200 \mathrm{mcd}$. The beam divergence is 10 degree. For detector, a silicon PIN photodiode SFH2030 (SIEMENS) was used ${ }^{10)}$. The diameter is $5 \mathrm{~mm}$. The LED and the photodiode were horizontally set on an optical axis face to face. The illuminance in the room was usual interior illumination (approximately $1500 \mathrm{kx}$ ).

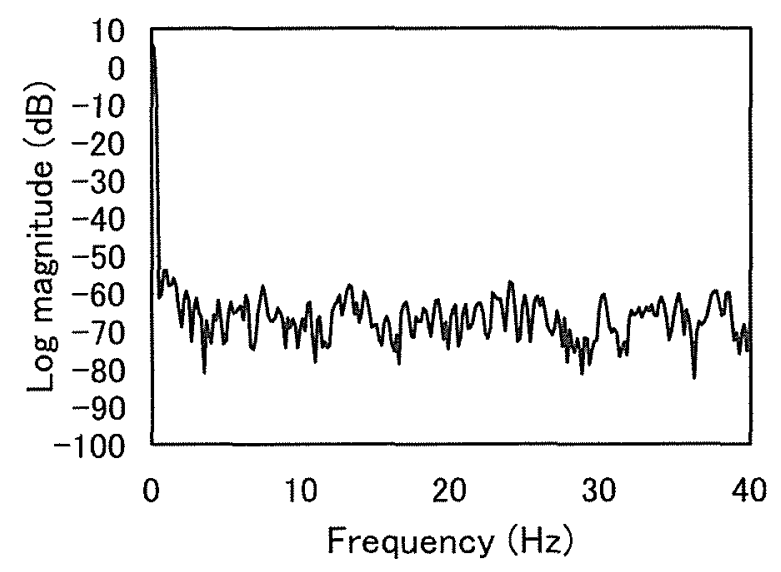

Figure 6 Power spectrum of LED output of improved



Figure 7 Power spectrum of LED output of reported method
The fluctuation could not be detected in the new method. Figure 6 shows the power spectrum of LED output of improved modulation technique. To compare the old and the new modulation techniques, the power spectrum of LED output by previously reported method was also measured (Figure 7). The component of low frequency was removed in Fig. 6.

\section{Luminous flux for communication}

The illuminance due to LED was also evaluated. When the distance between the LED and the photodiode was a few meters, the sound was reproduced with a quality similar to AM broadcasting. Figure 8 shows an example of transmitted and received signal of woman's narration with the distance of $4 \mathrm{~m}$.

The estimated illuminance due to the LED (9200 med) is $0.58 \mathrm{~lx}$ when the distance is $4 \mathrm{~m}$. This value is extremely small compared with the background illuminance. It means that the signal from LED is more than enough to transmit an audio signal when the lighting is replaced by LED.

The background illuminance does not affect the quality of received sound because the background illuminance does not have a component of high frequency.

For application in an office room as a lighting communication system, the coverage of communication was evaluated. We suppose a condition that LEDs are set on the ceiling and the receiver is put on a desk. While LEDs are used to light and transmit audio signal, the luminous flux received by the photodiode depends on the directions of the LED and the photodiode. It also depends on the distance between the LED and the photodiode. Figure 9 shows an example of arrangement of the LED and the photodiode. The luminous flux received by the photodiode, $\phi$, is expressed by

$$
\phi=\frac{S \times \cos \psi \times I(\theta)}{r^{2}}
$$



Figure 8 Transmitter signal and receiver signal 


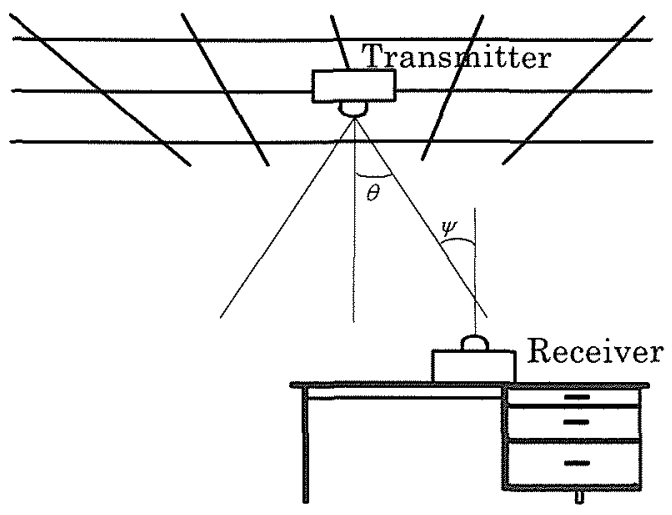

Figure 9 Example of arrangement of LED and photodiode

Here, $S$ is the received area of photodiode, $\psi$ is the angle of incidence, $\theta$ is the angle of radiation, $r$ is the distance between LED and photodiode, $I(\theta)$ is the luminous intensity of LED depending on the direction of the irradiation. If the LED is put looking nadir and the photodiode looks up zenith, $\theta$ is equal to $\psi$.

The received luminous flux by the photodiode was $1.1 \times 10^{-5} \mathrm{~lm}$ when the distance between the NSPW500BS LED and the SFH2030 photodiode was $4 \mathrm{~m}$. Therefore, we can receive the sound with the quality as shown in Fig. 8 if the received luminous flux is more than $1.1 \times 10^{-5} \mathrm{~lm}$. For example, if the vertical distance between NSPW500BS LED and SFH2030 photodiode is 2 $\mathrm{m}$, the angle of radiation is 10 degree, estimated luminous flux becomes $2.2 \times 10^{-5} \mathrm{~lm}$.

\section{Evaluation of interference}

We must evaluate the effects of interference for the office room applications using LEDs on the ceiling. If plural LEDs transmit same signal, the interference of signals due to path difference must be considered. The interference occurs if the phases of received signal as shown in Fig. 5 differ due to path difference.

The period of AM frequency is $1 \mu \mathrm{sec}$, when the frequency is $1 \mathrm{MHz}$. In general, the distance between lamps is a few meters in office room. If the path difference is $3 \mathrm{~m}$, the difference in time becomes $10 \mathrm{~ns}$, which is much smaller than the period of AM frequency. Therefore we can neglect the interference due to path difference.

In our method, LEDs can broadcast plural programs with different $\mathrm{AM}$ frequencies. Therefore, the interference of different AM frequencies should be evaluated. Actually sound could be received with worse quality at some different AM frequencies. The power spectrum at the range of AM frequency was numerically evaluated using following condition: the radio frequency is $600 \mathrm{kHz}$, the

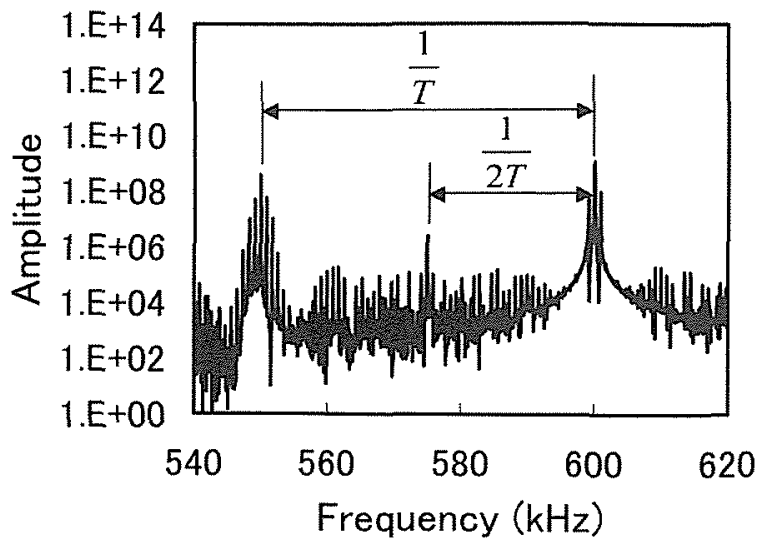

Figure 10 Simulated power spectrum of transmitted signal

sinusoidal waveform of $880 \mathrm{~Hz}$ was used for the audio signal and the period, $\mathrm{T}$ in Fig. 5, was $20 \mu$ sec. Figure 10 shows the simulated power spectrum of transmitted signal. The largest peak at $600 \mathrm{kHz}$ corresponds to the transmitted signal. There are two peaks corresponding to the audio signal at the both sides of main peak. There is peak at $550 \mathrm{kHz}$. The difference of frequency corresponds to $1 / \mathrm{T}$. There is a smaller peak at $575 \mathrm{kHz}$, too. The difference of frequency corresponds to $1 /(2 \mathrm{~T})$. Similar peaks exist at higher frequency region. Therefore these smaller peaks must be avoided when plural programs are broadcasted.

\section{Conclusions}

In this paper, an improved technique of transmitting audio signal using white LED was proposed. No fluctuation was sensed using the improved technique. The transmitter circuit was replaced by single microcomputer improving the technique.

It was verified that audio signal could be received with the quality similar to AM broadcasting when the received luminous flux is $1.1 \times 10^{-5} \mathrm{~lm}$.

We can think of other modulation methods for lighting communication. For example, Frequency-Shift Keying (FSK) can be used. Two frequency generators of different AM frequencies are prepared. The AM signals are switched according to the $\mathrm{T}_{\text {on }}$ and $\mathrm{T}_{\text {off }}$ in Fig. 5 and LED is modulated. However this method requires more complicated circuit and program than our method.

This technique can be used audio information in an office room to broadcast a news program, an emergency warning and a weather forecast, etc. It also can be used our method for guidance of several languages in a museum. It can be used in some places where electromagnetic wave cannot propagate or is prohibited in a hospital or in a tunnel, too. 


\section{References}

(1) Setomoto, T., Uchida, Y. and Taguchi, T.: Mluminance Characteristics of Energy-saving Luminaire with White-Light-Emitting Diode and a Solar-cell and Battery System for Street Lighting, J.IEIJ, Vol.85, No.8A, pp.577-584(2001).

(2) Taguchi, T:: "The Light for the 21st Century" National Project Based on White Light-Emitting Diode (LED) Lighting Technology, IEICE C, Vol. J84-C, No.11, pp.1040-1049(2001).

(3) Haruyama, S.: Visible Light Communication, IEICE Trans. Commun., Vol. J86-A, No.12, pp.1284-1291 (2003).

(4) Komine, T. and Nakagawa, M.: Fundamental Analysis for Visible-Light Communication System using LED Lights, IEEE Trans. Consum. Electron., Vol. 50, No. 1, pp.100-107(2004).
(5) Hashimoto, H. and Ogawa, A: Optical AM Wireless Communication System Using LEDs, Technical Report of IEICE, IT2003-86, pp.75-78(2004).

(6) Komine, T. and Nakagawa, M.: Integrated System of White LED Visible-Light Communication and PowerLine Communication, IEEE Trans. on Consumer Electronics, Vol.49 No.1, pp.71-79(2003).

(7) Kitano, S., Haruyama, S. and Nakagawa, M.: LED Road Illumination Communications System, Technical Report of IEICE, WBS2003-38, pp.37-40 (2003).

(8) Minato, A., Itaba, M., Ozawa, S. and Nakagawa, M.: Simple Visible-Light Transmission System Composed of PWM Modulation and AM Receiver, IEICE Trans. Commun., Vol. J88-B, No.12, pp.2390-2393(2005).

(9) ATtiny26 Data sheet (ATMEL, 2003).

(10) SFH2030 Data sheet (SIEMENS). 